\title{
Antifungal Quinoline Alkaloids from Waltheria indica
}

Sylvian Cretton, ${ }^{\dagger}$ Stéphane Dorsaz, ${ }^{\circledR}$ Antonio Azzollini, ${ }^{\dagger}$ Quentin Favre-Godal, ${ }^{\dagger}$ Laurence Marcourt, ${ }^{\dagger}$ Samad Nejad Ebrahimi, ${ }^{7}$ Francine Voinesco, ${ }^{¥}$ Emilie Michellod, ${ }^{¥}$ Dominique Sanglard ${ }^{\ddagger}$ Katia Gindro, ${ }^{\ddagger}$ Jean-Luc Wolfender, ${ }^{\dagger}$ Muriel Cuendet, ${ }^{\dagger}$ and Philippe Christen ${ }^{*}{ }^{\dagger}$

${ }^{\dagger}$ School of Phar maceutical Sciences, Univers ity of Geneva, University of Lausanne, Quai ErnestAnsermet 30, 1211 Geneva 4, Switzerland

"Institute of Microbiology, University of Lausanne and University Hospital Center, Laus conne, Switzerland

'Department of Phytochemistry, Medicinal Plants and Drugs Research Institute, Shahid Beheshti University, G. C., Evin, Tehran, Iran

Agroscope, Ins titute for Plant Production Sciences IPS, Mycology and B iotechnology, Route de Duiller 50, CP 1012, 1260 Nyon, Switzerland

"To whom correspondence should be addressed. Tel.: +4122379 65 61. Fax: +412237933 99. Email: philippe.christen@unige.ch 
S1. $1 \mathrm{H}$ NMR (500 MHz, CD3OD) spectrum of Waltherione M (2)

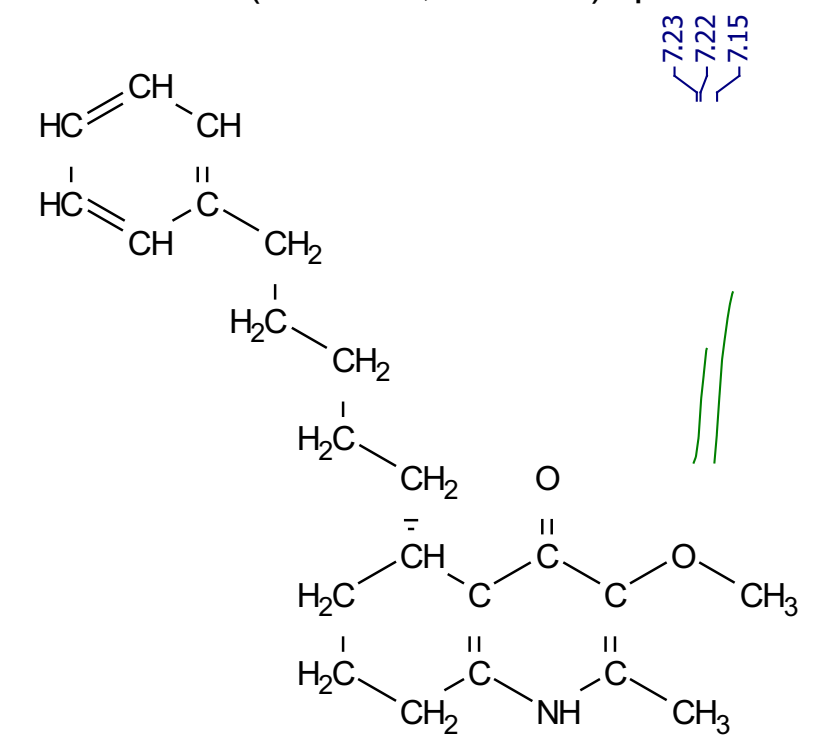

il

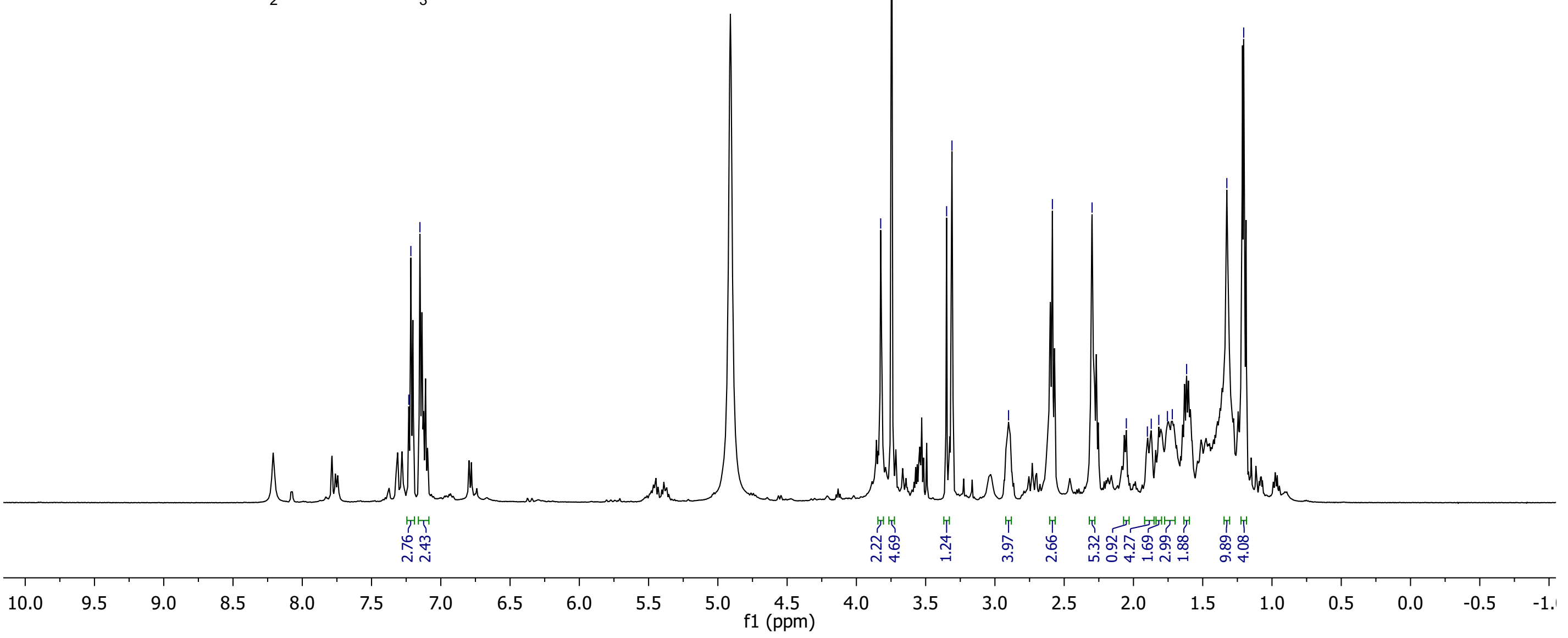


S2. $13 \mathrm{C}$ NMR (125 MHz, CD3OD) spectrum of Waltherione M (2) |

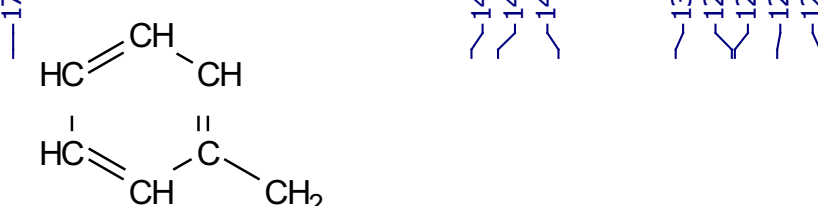<smiles>CC</smiles><smiles>[CH]C</smiles><smiles>C[CH][CH]CCOC</smiles>
$\mathrm{H}_{2} \mathrm{C}^{\mathrm{C}} \longrightarrow \mathrm{CH}_{2}^{-}{ }^{\prime \prime} \backslash \mathrm{NH}^{-}-\mathrm{C} \backslash \mathrm{CH}_{3}$

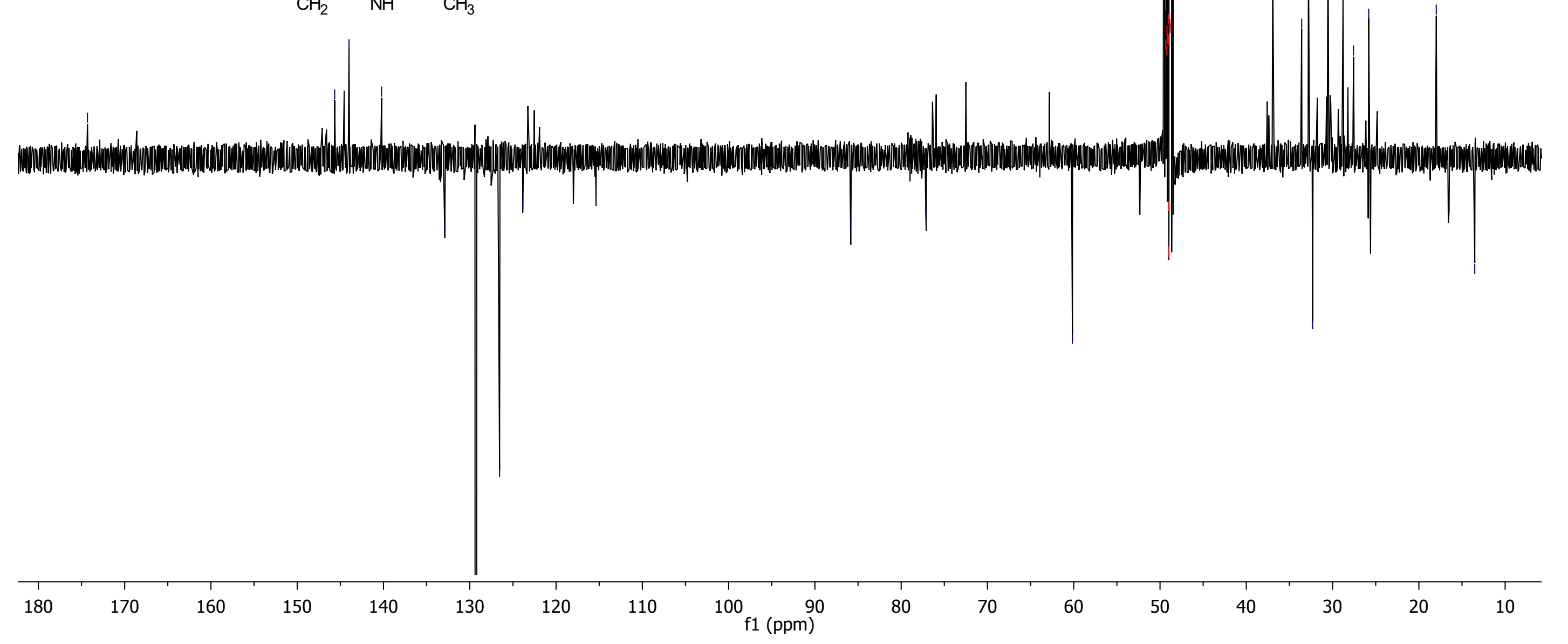


S3. $1 \mathrm{H}$ NMR (500 MHz, CD3OD) spectrum of Waltherione $\mathrm{N}(7)$<smiles>CCCCCCCC[C@H]1C=C(NC(C)=C(OC)C(=O)COC)[C@H](O)CC1</smiles>

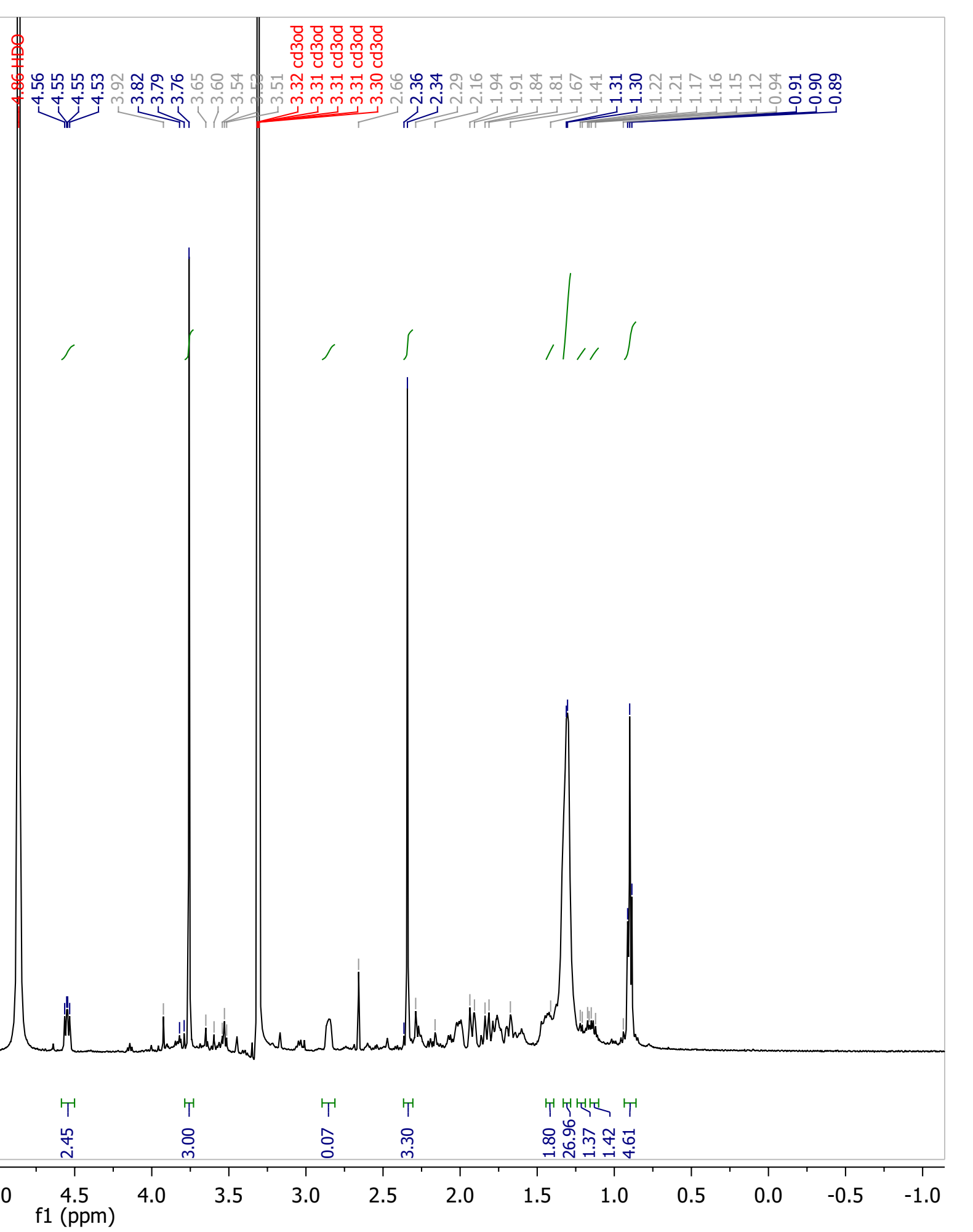




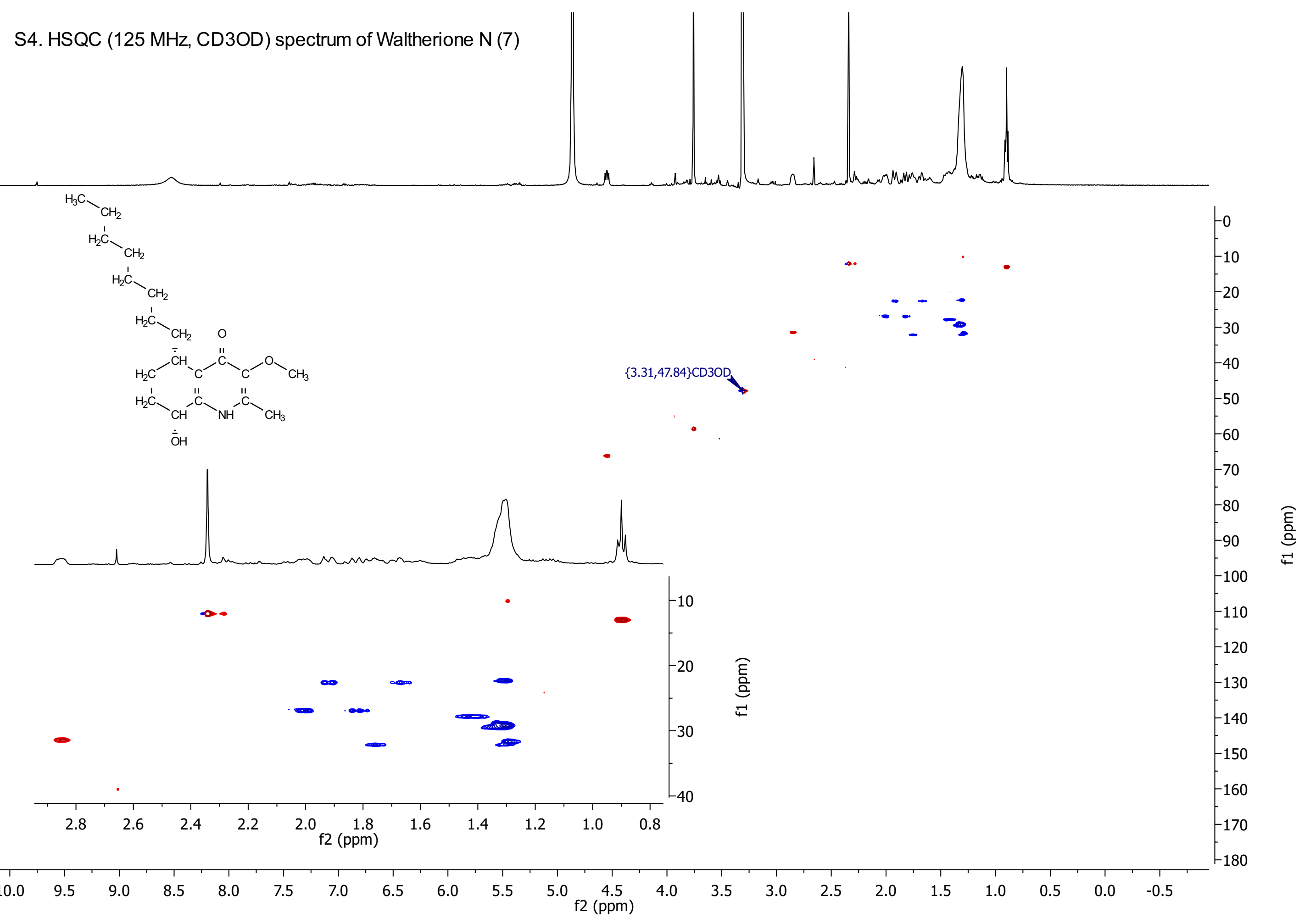


S6. 1H NMR (500 MHz, CD3OD) spectrum of Waltherione O (8)

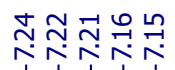<smiles>C#CC#C</smiles><smiles>CCC[C@H]1CCCc2[nH]c(CO)c(CO)c(=O)c21</smiles>

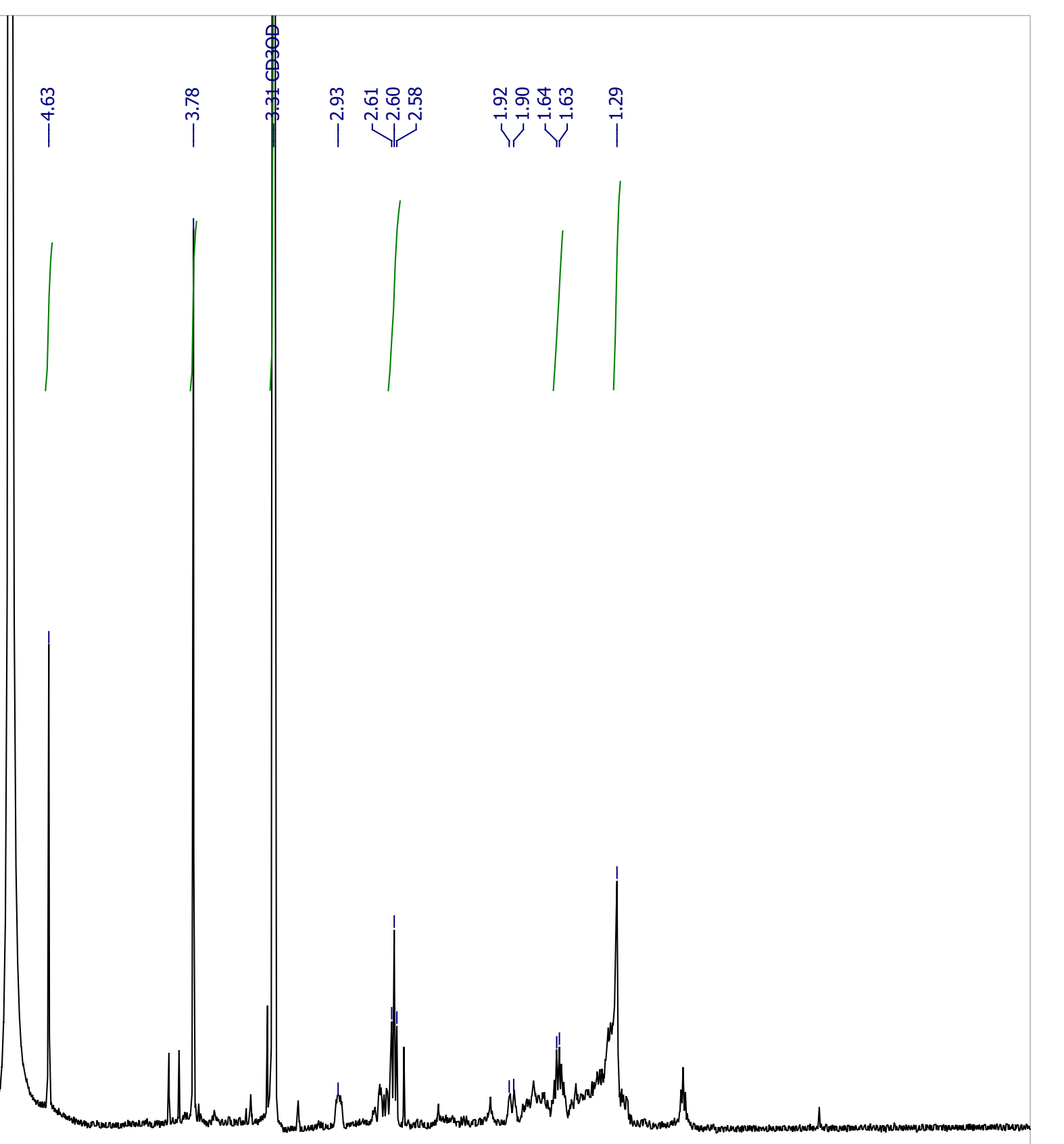

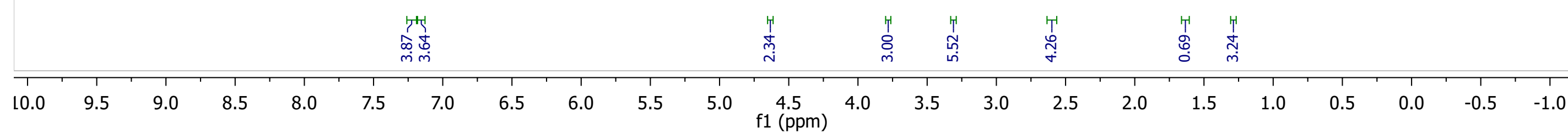


S7. HSQC (125 MHz, CD3OD) spectrum of Waltherione O (8)

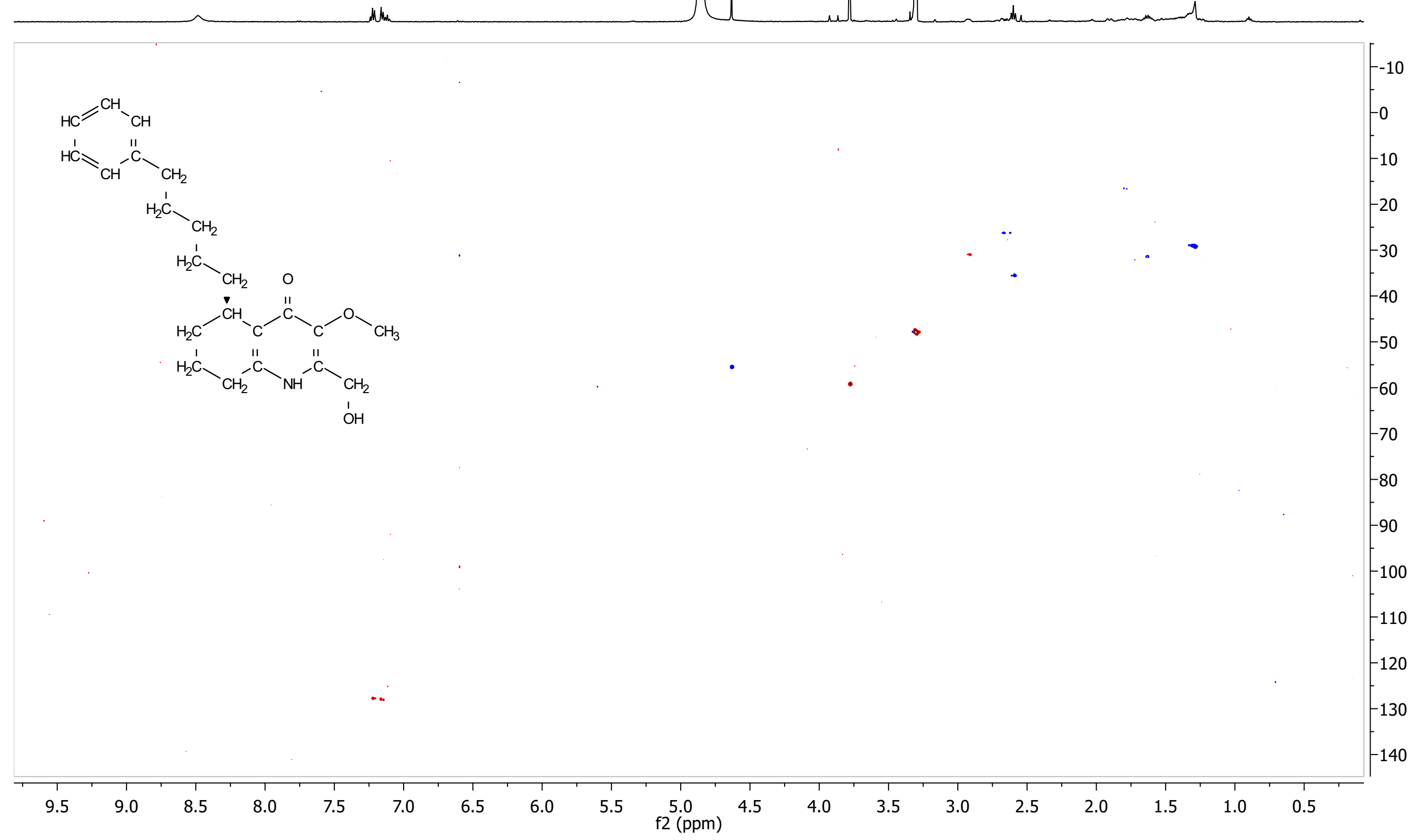



S9. $1 \mathrm{H} \mathrm{NMR}(500 \mathrm{MHz}, \mathrm{CD} 3 \mathrm{OD})$ spectrum of Waltherione $\mathrm{P}(10)$

$\mathrm{HC}=\mathrm{CH}_{-} \mathrm{CH}$

ำ 꼭

$\mathrm{HC}^{\prime}{ }_{\mathrm{CH}}{ }^{\prime \prime}{ }^{\prime \prime} \backslash \mathrm{CH}_{2}$

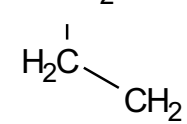

$\mathrm{H}_{2}^{\prime} \mathrm{C}^{\mathrm{C}}{ }_{\mathrm{CH}_{2}} \mathrm{O}$

$\mathrm{H}_{2} \mathrm{C}^{-} \stackrel{\overline{\mathrm{C}}}{\mathrm{H}_{-}} \mathrm{C}^{-}-{ }^{\prime \prime} \backslash \mathrm{C}^{-}-\mathrm{O}_{\mathrm{CH}_{3}}$

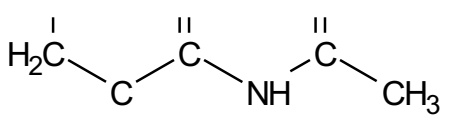

"

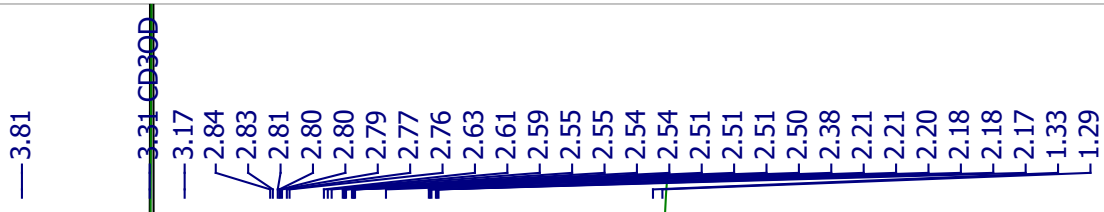
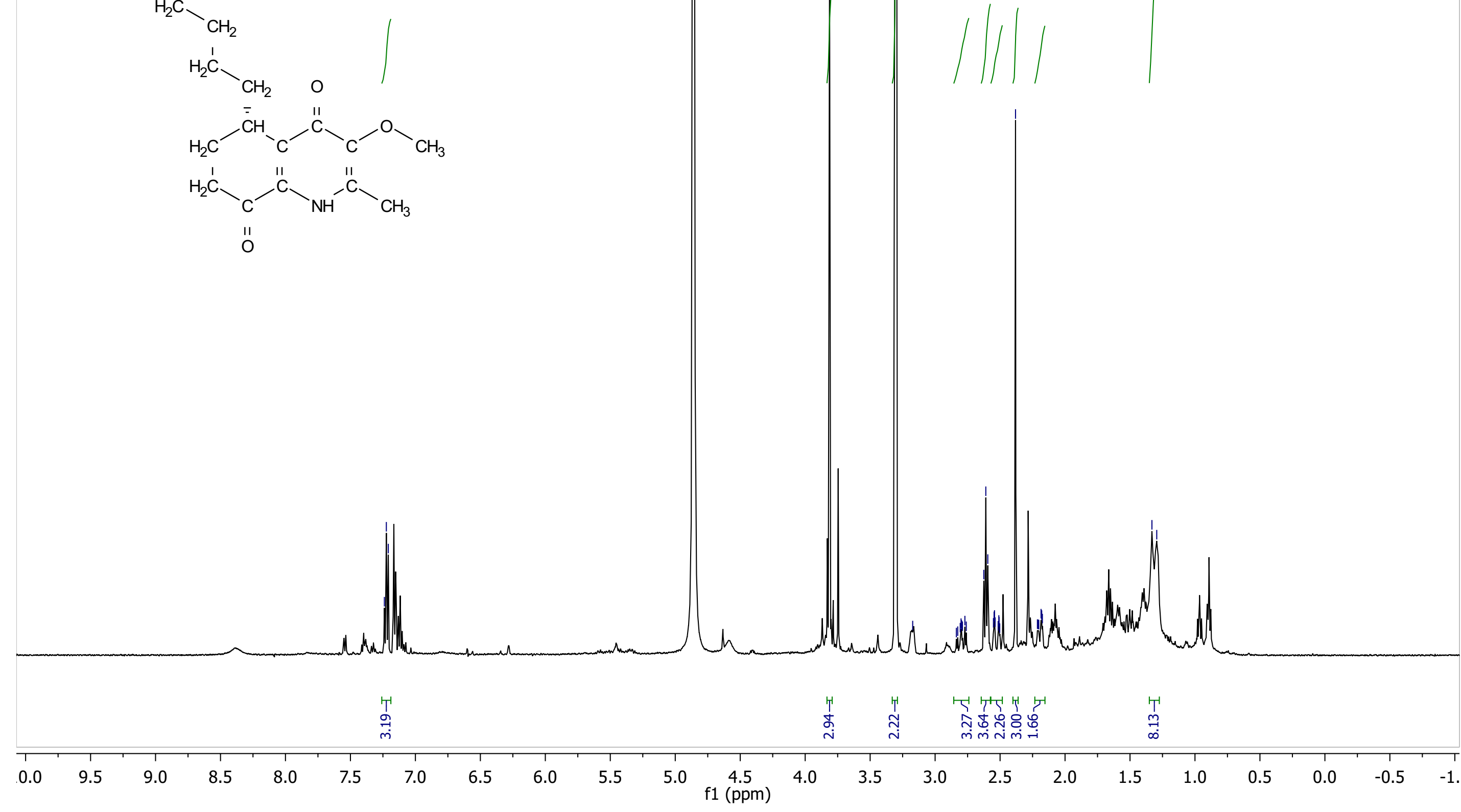


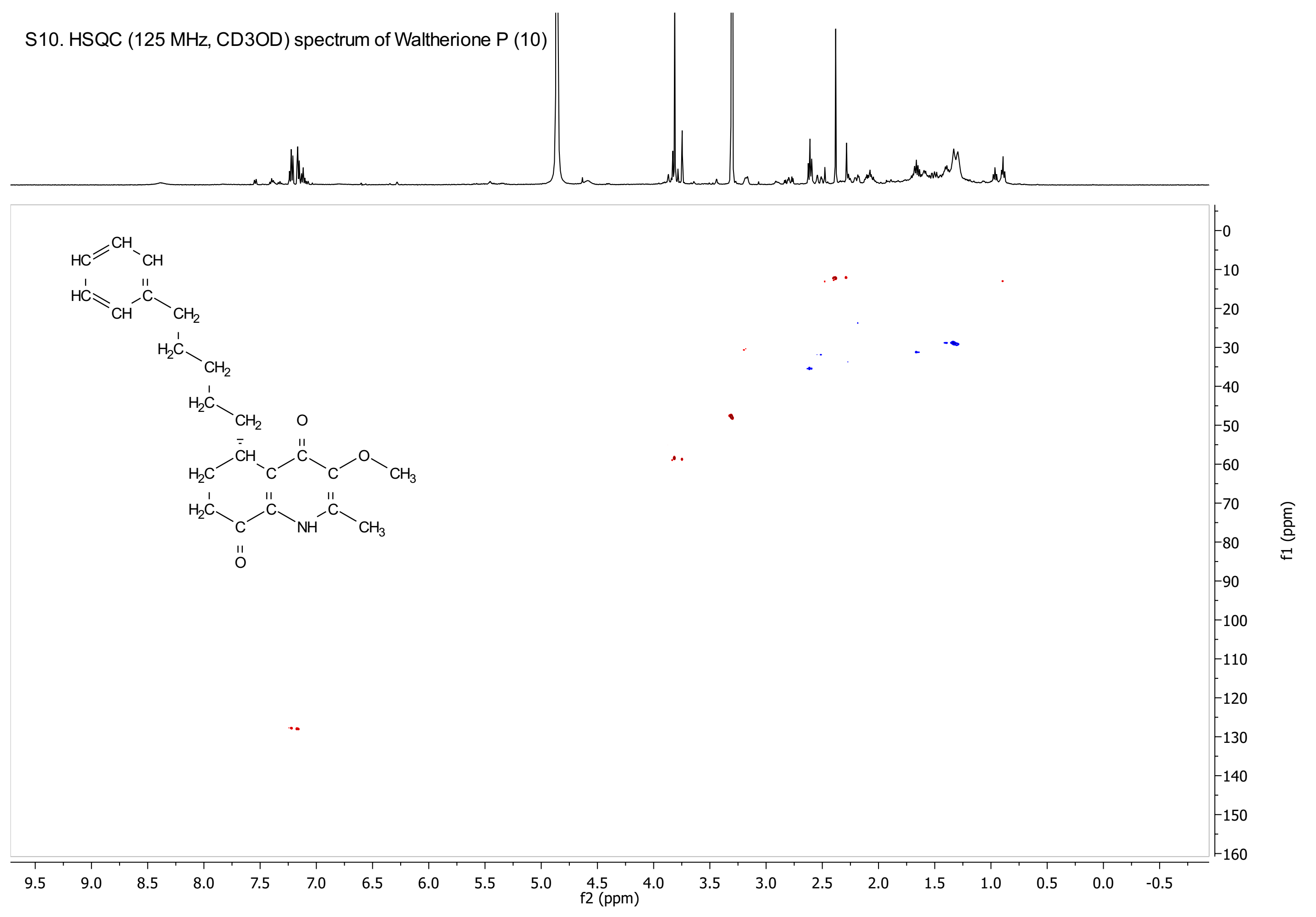




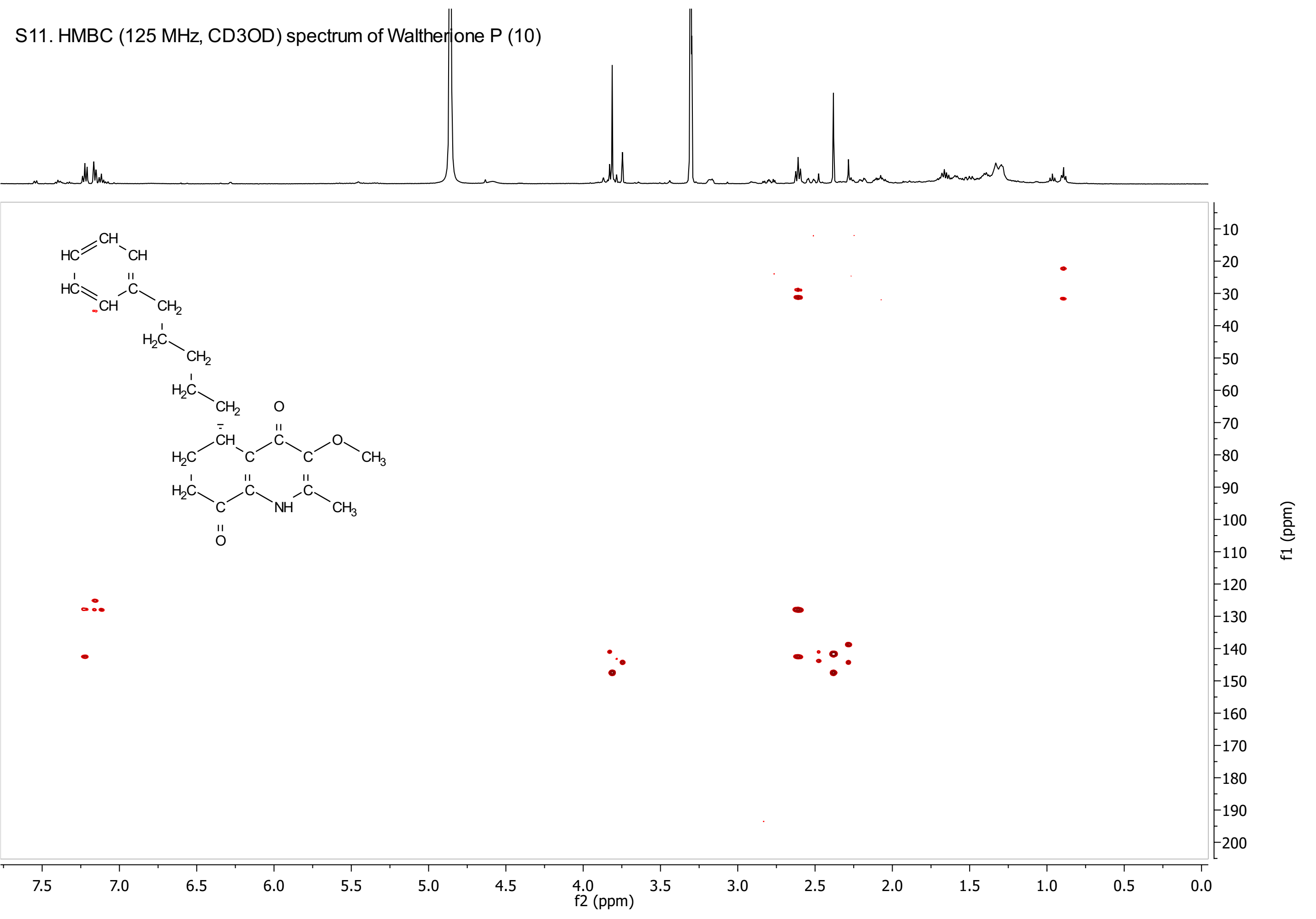


S12. $1 \mathrm{H}$ NMR (500 MHz, CD3OD) spectrum of Waltherione Q (11)

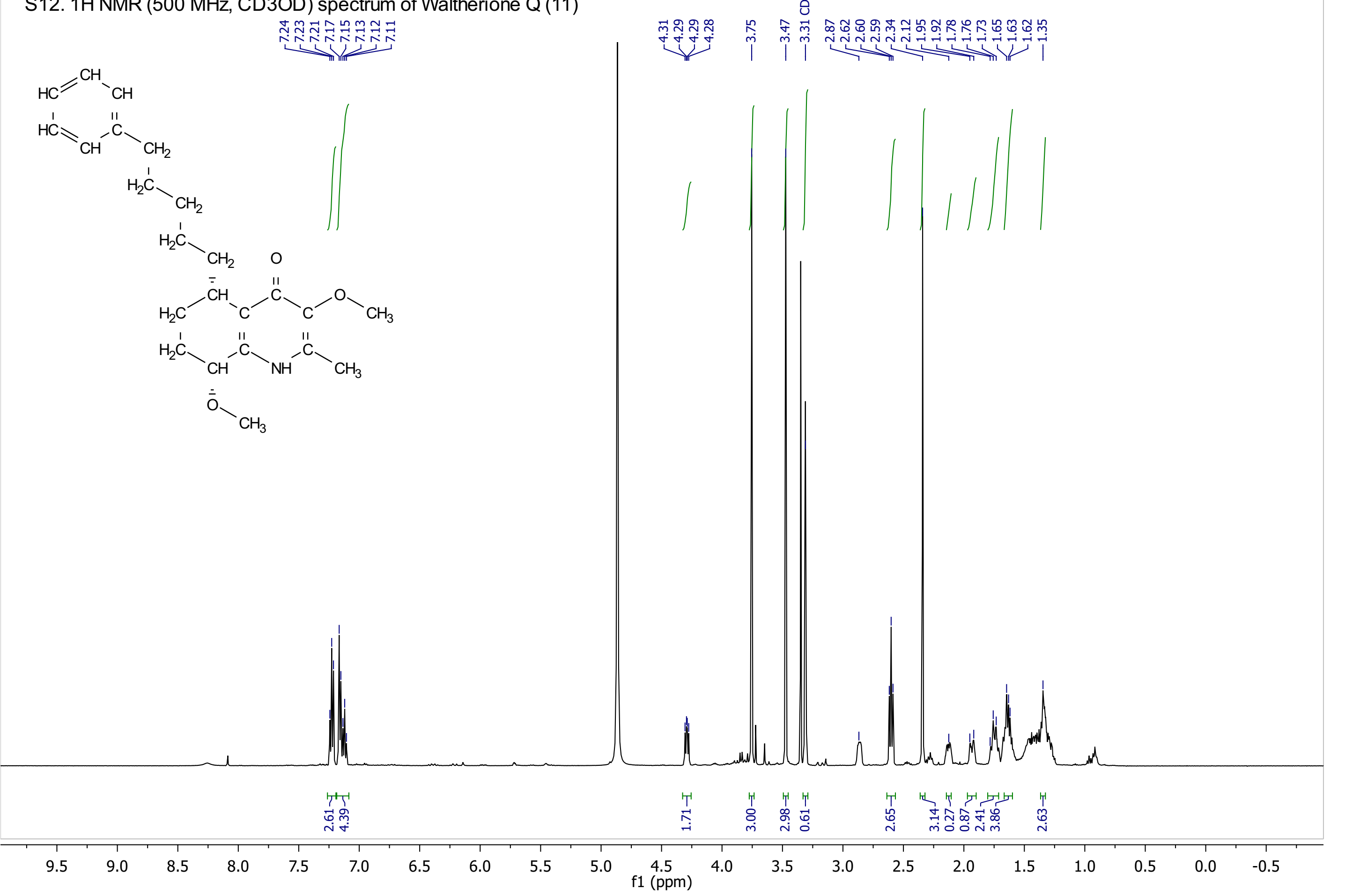




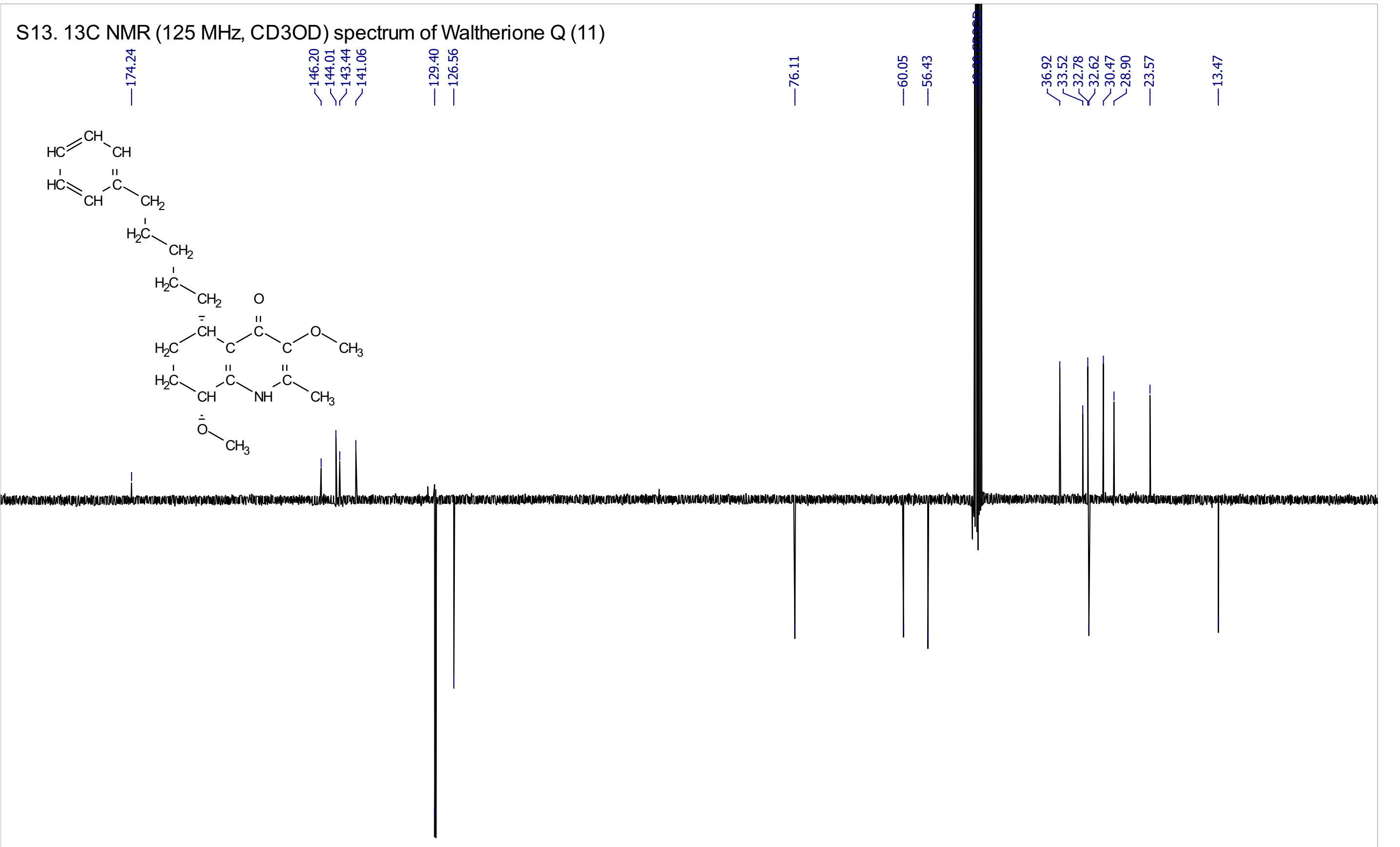

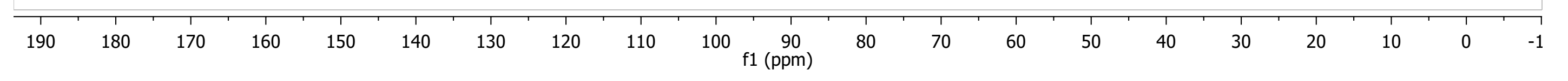

